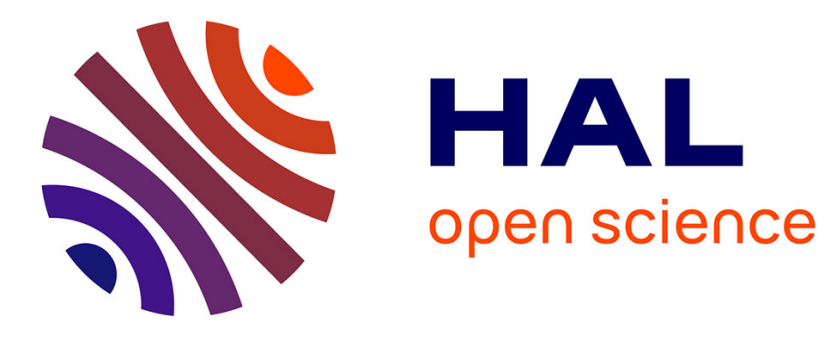

\title{
Constrained Clustering using SAT
}

Jean-Philippe Metivier, Patrice Boizumault, Bruno Crémilleux, Medhi Khiari, Samir Loudni

\section{To cite this version:}

Jean-Philippe Metivier, Patrice Boizumault, Bruno Crémilleux, Medhi Khiari, Samir Loudni. Constrained Clustering using SAT. 11th Int. Symposium on Intelligent Data Analysis (IDA 2012), Oct 2012, Helsinki, Finland. pp.207-218. hal-01023070

\section{HAL Id: hal-01023070 https://hal.science/hal-01023070}

Submitted on 15 Jul 2014

HAL is a multi-disciplinary open access archive for the deposit and dissemination of scientific research documents, whether they are published or not. The documents may come from teaching and research institutions in France or abroad, or from public or private research centers.
L'archive ouverte pluridisciplinaire HAL, est destinée au dépôt et à la diffusion de documents scientifiques de niveau recherche, publiés ou non, émanant des établissements d'enseignement et de recherche français ou étrangers, des laboratoires publics ou privés. 


\title{
Constrained Clustering Using SAT
}

\author{
Jean-Philippe Métivier, Patrice Boizumault, Bruno Crémilleux, \\ Mehdi Khiari, and Samir Loudni \\ University of Caen Basse-Normandie - GREYC (CNRS UMR 6072) \\ Campus II, Côte de Nacre, 14000 Caen - France \\ \{firstname.lastname\}@unicaen.fr
}

\begin{abstract}
Constrained clustering - finding clusters that satisfy userspecified constraints - aims at providing more relevant clusters by adding constraints enforcing required properties. Leveraging the recent progress in declarative and constraint-based pattern mining, we propose an effective constraint-clustering approach handling a large set of constraints which are described by a generic constraint-based language. Starting from an initial solution, queries can easily be refined in order to focus on more interesting clustering solutions. We show how each constraint (and query) is encoded in SAT and solved by taking benefit from several features of SAT solvers. Experiments performed using MiniSat on several datasets from the UCI repository show the feasibility and the advantages of our approach.
\end{abstract}

\section{Introduction}

Clustering is one of the core problems in data mining. Clustering aims at partitioning data into groups (clusters) so that transactions occurring in the same cluster are similar but different from those appearing in other clusters [12]. The usual clustering problem is designed to find clusterings satisfying a nearest representative property while constrained clustering 319] aims at obtaining more relevant clusters by adding constraints enforcing several properties expressing background information on the problem at hand. Constraints deal with various types: (1) data objects' relationships (e.g., a set of objects must be (or not) in a same cluster [20]), (2) the description of the clusters (e.g., a cluster must have a minimal or a maximal size [2]), (3) both objects and clusters (e.g., a given object must be in a given cluster), (4) the characteristics of the clustering (e.g., the number of clusters),... Traditional clustering algorithms do not provide effective mechanisms to make use of this information. The goal of this paper is to propose a generic approach to fill this gap.

Recently, several works have investigated relationships between data mining and constraint programming $(\mathrm{CP})$ to revisit data mining tasks in a declarative and generic way 6 6 14 15]. The user models a problem and expresses his queries by specifying what constraints need to be satisfied. The process greatly facilitates the search of knowledge and models such as clustering. The approach is enforced by the use of a constraint-based language [17]: it is sufficient to change 
the specification in term of constraints to address different pattern mining problems. In the spirit of this promising avenue, we propose an effective constrained clustering approach handling a large set of constraints.

The paper brings the following contributions. First, we use the declarative modeling principle of CP to define a constrained clustering approach taking into account a large set of constraints on objects, a description of the clusters and the clustering process itself. By nature, clustering proceeds by iteratively refining queries until a satisfactory solution is found. Our method integrates in a natural way this stepwise refinement process based on the queries in order to focus on more interesting clustering solutions. Contrary to very numerous clustering methods that use heuristics or greedy algorithms, our method is complete. Second, we define an efficient SAT encoding which integrates features of SAT solvers (e.g., binary clauses, unit propagation, sorting networks) to solve the queries. Finally, an experimental study using MiniSat shows the feasibility and the effectiveness of our method on several datasets from the UCI repository.

Section 2 provides the background on the constraint-based language. Section 3 describes our method on constrained clustering with examples of constraints coming from the background information of the problem at hand. Section 4 addresses the point of how queries and constraints of the language are encoded and solved with SAT. Section 5 shows the effectiveness of our approach through several experiments. Section 6 presents related work.

\section{Background: Constraint-Based Language}

The constraint programming methodology is by nature declarative. It explains why studying relationships between CP and data mining has received a considerable attention to go towards generic and declarative data mining methods 6 14 15. This section sketches our constraint-based language that enables us to specify in term of constraints different pattern mining problems [17]. This language forms the first step of our constrained clustering method proposed in Section 3. In the remainder of this section, we only focus on primitives of the language that will be used in this paper.

Let $\mathcal{I}$ be a set of $n$ distinct literals called items, an itemset (or pattern) is a non-null subset of $\mathcal{I}$. The language of itemsets corresponds to $\mathcal{L}_{\mathcal{I}}=2^{\mathcal{I}} \backslash \emptyset$. A transactional dataset $\mathcal{T}$ is a multi-set of $m$ itemsets of $\mathcal{L}_{\mathcal{I}}$. Each itemset, usually called a transaction or object, is a database entry. For instance, Table 1 gives a transactional dataset $\mathcal{T}$ with $m=11$ transactions $t_{1}, \ldots, t_{11}$ described by $n=10$ items. This toy dataset is inspired by the Zoo dataset from the UCI repository.

Terms are built from constants, variables, operators, and function symbols. Constants are either numerical values, or patterns, or transactions. Variables, noted $X_{j}$, for $1 \leq j \leq k$, represent the unknown patterns (or clusters). Operators can be set ones (as $\cap, \cup, \backslash$ ) or numerical ones (as,,$+- \times, /$ ). Built-in function symbols involve one or several terms:

- cover $\left(X_{j}\right)=\left\{t \mid t \in \mathcal{T}, X_{j} \subseteq t\right\}$ set of transactions covered by $X_{j}$.

- $\operatorname{freq}\left(X_{j}\right)=\left|\left\{t \mid t \in \mathcal{T}, X_{j} \subseteq t\right\}\right|$ is the frequency of pattern $X_{j}$. 
$-\operatorname{size}\left(X_{j}\right)=\left|\left\{i \mid i \in \mathcal{I}, i \in X_{j}\right\}\right|$ is the size of pattern $X_{j}$.

- overlapItems $\left(X_{i}, X_{j}\right)=\left|X_{i} \cap X_{j}\right|$ is the number of items shared by both $X_{i}$ and $X_{j}$.

- overlapTransactions $\left(X_{i}, X_{j}\right)=\left|\operatorname{cover}\left(X_{i}\right) \cap \operatorname{cover}\left(X_{j}\right)\right|$ is the number of transactions covered by both $X_{i}$ and $X_{j}$.

Constraints are relations over terms that can be satisfied or not. There are three kinds of built-in constraints: numerical constraints (like $<, \leq,=, \neq, \geq,>$ ), set constraints (like $=, \neq, \in, \notin, \subset, \subseteq$ ), and dedicated constraints like:

- isNotEmpty $\left(X_{j}\right)$ is satisfied iff $X_{j} \neq \emptyset$

- coverTransactions $\left(\left[X_{1}, \ldots, X_{k}\right]\right)$ is satisfied iff each transaction is covered by at least one pattern $\left(\bigcup_{1 \leq i \leq k} \operatorname{cover}\left(X_{i}\right)=\mathcal{T}\right)$

- noOverlapTransactions $\left(\left[\bar{X}_{1}, \ldots, X_{k}\right]\right)$ is satisfied iff all $i, j$ s.t. $1 \leq i<j \leq k$, $\operatorname{cover}\left(X_{i}\right) \cap \operatorname{cover}\left(X_{j}\right)=\emptyset$

- coverItems $\left(\left[X_{1}, \ldots, X_{k}\right]\right)$ is satisfied iff each item belongs to at least one pattern $\left(\cup_{1 \leq i \leq k} X_{i}=\mathcal{I}\right)$

- noOverlapItems $\left(\left[X_{1}, \ldots, X_{k}\right]\right)$ is satisfied iff $\forall i, j$ s.t. $1 \leq i<j \leq k, X_{i} \cap X_{j}=\emptyset$

- canonical $\left(\left[X_{1}, \ldots, X_{k}\right]\right)$ is satisfied iff for all $i$ s.t. $1 \leq i<k$, pattern $X_{i}$ is less than pattern $X_{i+1}$ with respect to the lexicographic order.

Finally, a query is a conjunction of constraints as illustrated in the next section.

Table 1. Animal dataset

\begin{tabular}{lc|cccccccccc}
\multicolumn{1}{l|}{ Species } & trans & \multicolumn{1}{c}{ Fur } & Feather Scale Milk & Egg Beak & Bone & Meat & Grass Fish \\
\hline Cat & $t_{1}$ & 1 & 0 & 0 & 1 & 0 & 0 & 1 & 1 & 0 & 1 \\
Cow & $t_{2}$ & 1 & 0 & 0 & 1 & 0 & 0 & 1 & 0 & 1 & 0 \\
Crow & $t_{3}$ & 0 & 1 & 0 & 0 & 1 & 1 & 1 & 1 & 1 & 1 \\
Daulphin & $t_{4}$ & 0 & 0 & 0 & 1 & 0 & 0 & 1 & 0 & 0 & 1 \\
Dog & $t_{5}$ & 1 & 0 & 0 & 1 & 0 & 0 & 1 & 1 & 0 & 0 \\
Goose & $t_{6}$ & 0 & 1 & 0 & 0 & 1 & 1 & 1 & 0 & 1 & 0 \\
Platypus & $t_{7}$ & 1 & 0 & 0 & 1 & 1 & 1 & 1 & 1 & 0 & 0 \\
Salmon & $t_{8}$ & 0 & 0 & 1 & 0 & 1 & 0 & 0 & 0 & 1 & 1 \\
Shark & $t_{9}$ & 0 & 0 & 0 & 0 & 0 & 0 & 0 & 1 & 0 & 1 \\
Trout & $t_{10}$ & 0 & 0 & 1 & 0 & 1 & 0 & 0 & 0 & 1 & 0 \\
Vulture & $t_{11}$ & 0 & 1 & 0 & 0 & 1 & 1 & 1 & 1 & 0 & 1 \\
\hline
\end{tabular}

\section{Constrained Clustering: Modeling}

\subsection{Introduction: Modeling a Clustering Query}

A clustering problem can be thought of as a scenario in which a user wishes to obtain a partition $\pi_{T}=\left(X_{1}, \ldots, X_{k}\right)$ of a dataset $\mathcal{T}$, containing $m$ objects, into $k$ (non-empty) clusters. A clustering problem intrinsically owns a lot of symmetrical solutions: any permutation of $\pi_{T}$ is a solution. The canonical $\left(\left[X_{1}, \ldots, X_{k}\right]\right)$ constraint is used to avoid symmetrical solutions. 
So, we can define the isClustering $\left(\left[X_{1}, \ldots, X_{k}\right]\right)$ constraint:

$$
\text { isClustering }\left(\left[X_{1}, \ldots, X_{k}\right]\right) \equiv\left\{\begin{array}{l}
\wedge_{1 \leq i \leq k} \text { isNotEmpty }\left(X_{i}\right) \wedge \\
\text { coverTransactions }\left(\left[X_{1}, \ldots, X_{k}\right]\right) \wedge \\
\text { noDverlapTransactions }\left(\left[X_{1}, \ldots, X_{k}\right]\right) \wedge \\
\text { canonical }\left(\left[X_{1}, \ldots, X_{k}\right]\right)
\end{array}\right.
$$

\subsection{Integrating Background Information in the Clustering Process}

In many application domains, background information on the domain and/or dataset is often available and the data analyst would like to integrate it in the process to improve the clustering results. Such a knowledge is usually expressed as transaction-level constraints (like the mustLink and cannotLink constraints 20], and as cluster-level constraints (like Maximum Diameter and Minimum Separation contraints [78]).

We start by describing how these information can be modeled thanks to the constraint-based language (see Section 2). Then, we show how our method allow to combine them to achieve more relevant queries. Let $\mathcal{T}$ be a dataset of $m$ transactions. Let $d\left(t_{1}, t_{2}\right)$ be a distance over transactions.

Transaction-Level Constraints consist in mustLink and cannotLink constraints 20]:

- mustLink $\left(t_{1}, t_{2}\right)$ ensures that transactions $t_{1}$ and $t_{2}$ belong to the same cluster.

- cannotLink $\left(t_{1}, t_{2}\right)$ ensures that transactions $t_{1}$ and $t_{2}$ do not belong to the same cluster.

Cluster-Level Constraints. The diameter of a cluster $X_{j}$ is the maximum distance between a pair of transactions in $X_{j}$ [7]8]. The cluster-level constraint maximum diameter requires that the diameter of any cluster be at most a given value $\alpha$. To achieve this, we must ensure that any pair of transactions $\left(t_{i}, t_{j}\right)$ with $d\left(t_{i}, t_{j}\right)>\alpha$ are in different clusters. So, for $1 \leq i<j \leq m$, if $d\left(t_{i}, t_{j}\right)>\alpha$ then the constraint cannotLink $\left(t_{i}, t_{j}\right)$ must be added.

The separation between two clusters $X_{i}$ and $X_{j}$ is the minimum distance between a pair of transactions, one from $X_{i}$ and the other from $X_{j}$. The clusterlevel constraint Minimum Separation requires that the separation between two clusters be at least a given value $\beta$. To achieve this, we must ensure that any pair of transactions $\left(t_{i}, t_{j}\right)$ with $d\left(t_{i}, t_{j}\right)<\beta$ are in the same cluster. So, for $1 \leq i<j \leq m$, if $d\left(t_{i}, t_{j}\right)<\beta$ then the constraint mustLink $\left(t_{i}, t_{j}\right)$ must be added. Cluster-level constraints can be combined together (query $q_{1}$ ).

$$
q_{1}\left(\left[X_{1}, \ldots, X_{k}\right]\right) \equiv\left\{\begin{array}{l}
\text { isClustering }\left(\left[X_{1}, \ldots, X_{k}\right]\right) \wedge \\
\wedge_{1 \leq i<j \leq m, d\left(t_{i}, t_{j}\right)<\beta} \text { mustLink }\left(t_{i}, t_{j}\right) \wedge \\
\wedge_{1 \leq i<j \leq m, d\left(t_{i}, t_{j}\right)>\alpha} \text { cannotLink }\left(t_{i}, t_{j}\right)
\end{array}\right.
$$

The method can be performed with any distance between transactions. For instance, when transactions are described with numerical values, a numerical distance such as the euclidian distance can be used. 
Seeding. Background information both on transactions and clusters is easily modeled in the same way. Let $t_{i_{0}}$ and $t_{j_{0}}$ be two transactions and $X_{j_{1}}$ a cluster. $t_{i_{0}}$ and $t_{j_{0}}$ must be (resp. must not be) in a same cluster is modeled by adding the constraint mustLink $\left(t_{i_{0}}, t_{j_{0}}\right)$ (resp. cannotLink $\left(t_{i_{0}}, t_{j_{0}}\right)$ ). $t_{i_{0}}$ must be (resp. must not be) in the cluster $X_{j_{1}}$ is modeled by adding the constraint $t_{i_{0}} \in X_{j_{1}}$ (resp. $t_{i_{0}} \notin X_{j_{1}}$ ).

\subsection{Stepwise Refinements for Clustering}

A major strength of our approach is to provide a simple and efficient way to declare and refine queries, that is usually the process conducted by a data analyst when he performs clustering tasks. Starting from an initial query (like $q_{1}$ ), the data analyst can express that he prefers solutions with a minimal size of the clusters, in which the sizes of clusters do not differ too much from each other, etc. In practice, the data analyst successively refines the query (deriving $q_{i+1}$ from $q_{i}$ ) until he considers that relevant information has been extracted. This stepwise refinement process is easily handled by our constrained clustering approach as illustrated below.

Removing Clusterings with Small Size Patterns. A clustering including at least one cluster $X_{i}$ of small size is not considered as useful because $X_{i}$ does not ensure enough similarity between transactions associated to $X_{i}$. Adding a minimal size threshold solves this drawback (query $q_{2}$ ).

$$
q_{2}\left(\left[X_{1}, \ldots, X_{k}\right]\right) \equiv\left\{\begin{array}{l}
\text { isClustering }\left(\left[X_{1}, \ldots, X_{k}\right]\right) \wedge \\
\wedge_{1 \leq i<j \leq m, d\left(t_{i}, t_{j}\right)<\beta} \operatorname{mustLink}\left(t_{i}, t_{j}\right) \wedge \\
\wedge_{1 \leq i<j \leq m, d\left(t_{i}, t_{j}\right)>\alpha} \operatorname{cannotLink}\left(t_{i}, t_{j}\right) \wedge \\
\wedge_{1 \leq i \leq k} \operatorname{size}\left(X_{i}\right) \geq \delta
\end{array}\right.
$$

Balanced Clustering. Clustering solutions in which the sizes of clusters do not differ too much from each other are generally preferred. For any pair of clusters $\left(X_{i}, X_{j}\right)$, their difference of sizes must be lower than a threshold $\Delta \times m$ where $\Delta$ is a percentage (query $q_{3}$ ).

$$
q_{3}\left(\left[X_{1}, \ldots, X_{k}\right]\right) \equiv\left\{\begin{array}{l}
\text { isClustering }\left(\left[X_{1}, \ldots, X_{k}\right]\right) \wedge \\
\wedge_{1 \leq i<j \leq m, d\left(t_{i}, t_{j}\right)<\beta} \operatorname{mustLink}\left(t_{i}, t_{j}\right) \wedge \\
\wedge_{1 \leq i<j \leq m, d\left(t_{i}, t_{j}\right)>\alpha} \operatorname{cannotLink}\left(t_{i}, t_{j}\right) \wedge \\
\wedge_{1 \leq i<j \leq k}\left|\operatorname{size}\left(X_{i}\right)-\operatorname{size}\left(X_{j}\right)\right| \leq \Delta \times m
\end{array}\right.
$$

\subsection{An Example of Stepwise Refinements}

Let $k=3$ and $d\left(t_{1}, t_{2}\right)$ be the Hamming distance between transaction $t_{1}$ and transaction $t_{2}$. Using the dataset $\mathcal{T}$ described in Table 1 query $q_{1}^{\prime}$ provides 966 solutions for $\alpha=9$ and $\beta=1$. By refining these thresholds (decreasing the maximal diameter to $\alpha=8$ and enlarging the minimal separation to $\beta=2$ ), there remain four solutions (see Table 2).

$$
q_{1}^{\prime}\left(\left[X_{1}, X_{2}, X_{3}\right]\right) \equiv\left\{\begin{array}{l}
\text { isClustering }\left(\left[X_{1}, X_{2}, X_{3}\right]\right) \wedge \\
\wedge_{1 \leq i<j \leq m, d\left(t_{i}, t_{j}\right)<\beta} \operatorname{mustLink}\left(t_{i}, t_{j}\right) \wedge \\
\wedge_{1 \leq i<j \leq m, d\left(t_{i}, t_{j}\right)>\alpha} \operatorname{cannotLink}\left(t_{i}, t_{j}\right)
\end{array}\right.
$$


Table 2. Set of clusterings for $q_{1}^{\prime}(\alpha=8$ and $\beta=2)$

\begin{tabular}{r|l|l|l} 
Sol. & $X_{1}$ & $X_{2}$ & $X_{3}$ \\
\hline$s_{1}$ & $\left\{t_{1}, t_{2}, t_{4}, t_{5}, t_{7}\right\}$ & $\left\{t_{3}, t_{6}, t_{8}, t_{10}, t_{11}\right\}$ & $\left\{t_{9}\right\}$ \\
$s_{2}$ & $\left\{t_{1}, t_{2}, t_{4}, t_{5}, t_{7}\right\}$ & $\left\{t_{3}, t_{6}, t_{11}\right\}$ & $\left\{t_{8}, t_{9}, t_{10}\right\}$ \\
$s_{3}$ & $\left\{t_{1}, t_{2}, t_{4}, t_{5}, t_{7}\right\}$ & $\left\{t_{3}, t_{6}, t_{9}, t_{11}\right\}$ & $\left\{t_{8}, t_{10}\right\}$ \\
$s_{4}$ & $\left\{t_{1}, t_{2}, t_{4}, t_{5}, t_{7}, t_{9}\right\}$ & $\left\{t_{3}, t_{6}, t_{11}\right\}$ & $\left\{t_{8}, t_{10}\right\}$ \\
\hline
\end{tabular}

Clusters with a small size (e.g., $\left\{t_{9}\right\}$ in solution $s_{1}$ ) are considered irrelevant. By adding $\delta=2$ as a minimal cluster size threshold, we get query $q_{2}^{\prime}$ and there remains three solutions $\left(s_{2}, s_{3}\right.$, and $s_{4}$, see Table2).

$$
q_{2}^{\prime}\left(\left[X_{1}, X_{2}, X_{3}\right]\right) \equiv\left\{\begin{array}{l}
q_{1}^{\prime}\left(\left[X_{1}, X_{2}, X_{3}\right]\right) \wedge \\
\bigwedge_{1 \leq i \leq 3} \operatorname{size}\left(X_{i}\right) \geq \delta
\end{array}\right.
$$

The user may want to indicate that the shark $\left(t_{9}\right)$ must be in the same cluster as the salmon $\left(t_{8}\right)$. This is done with a mustlink constraint. The solution $s_{2}$ is then the unique solution for the query $q_{3}^{\prime}$ where the $k=3$ clusters respectively denote mammals, birds and fish.

$$
q_{3}^{\prime}\left(\left[X_{1}, X_{2}, X_{3}\right]\right) \equiv\left\{\begin{array}{l}
q_{2}^{\prime}\left(\left[X_{1}, X_{2}, X_{3}\right]\right) \wedge \\
\text { mustlink }\left(t_{8}, t_{9}\right)
\end{array}\right.
$$

\subsection{Other Clustering Problems}

In the same way, it is easy to express other clustering problems 5] such as soft clustering and co-clustering, the latter being well-used in bioinformatics for exploring gene expression data. Soft clustering is a relaxed version of the clustering where small overlaps on transactions (less than a threshold $\delta_{T}$ ) are allowed.

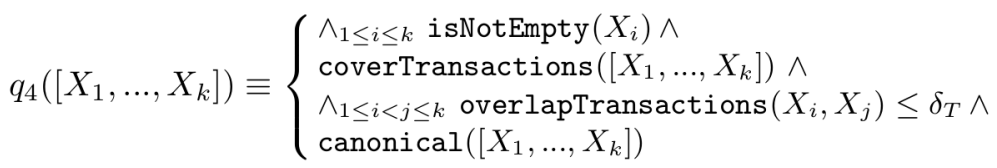

Co-clustering consists in finding $k$ clusters covering both the set of transactions and the set of items, without any overlap on transactions or on items.

$$
q_{5}\left(\left[X_{1}, \ldots, X_{k}\right]\right) \equiv\left\{\begin{array}{l}
\text { isClustering }\left(\left[X_{1}, \ldots, X_{k}\right]\right) \wedge \\
\text { coverItems }\left(\left[X_{1}, \ldots, X_{k}\right]\right) \wedge \\
\text { noDverlapItems }\left(\left[X_{1}, \ldots, X_{k}\right]\right)
\end{array}\right.
$$

Soft co-clustering is a relaxed version of the co-clustering, allowing small overlaps on transactions (less than $\delta_{T}$ ) and on items (less than $\delta_{I}$ ).

$$
q_{6}\left(\left[X_{1}, \ldots, X_{k}\right]\right) \equiv\left\{\begin{array}{l}
\wedge_{1 \leq i \leq k} \text { isNotEmpty }\left(X_{i}\right) \wedge \\
\text { coverTransactions }\left(\left[X_{1}, \ldots, X_{k}\right]\right) \wedge \\
\wedge_{1 \leq i<j \leq k} \text { overlapTransactions }\left(X_{i}, X_{j}\right) \leq \delta_{T} \wedge \\
\text { coverItems }\left(\left[X_{1}, \ldots, X_{k}\right]\right) \wedge \\
\wedge_{1 \leq i<j \leq k} \text { overlapItems }\left(X_{i}, X_{j}\right) \leq \delta_{I} \wedge \\
\text { canonical }\left(\left[X_{1}, \ldots, X_{k}\right]\right)
\end{array}\right.
$$




\section{Constrained Clustering: SAT Encoding}

\subsection{A Short Overview of SAT Solvers}

Satisfiability (SAT) is the problem of determining if the variables of a given boolean formula can be assigned in such a way as to make the formula be evaluated to True. A formula is in conjunctive normal form $(\mathrm{CNF})$ if it is a conjunction of clauses, where a clause is a disjunction of literals.

SAT solvers perform filtering using unit propagation. If a CNF $\mathcal{F}$ contains a unit clause (composed of a single literal $l$ ), then $\mathcal{F}$ is satisfied, if and only if, $l$ is assigned to True. So every clause containing $l$ can be removed and $\neg l$ can be deleted in every clause it occurs. Binary clauses are well suited for unit propagation. If one of its two literals is assigned, a binary clause is either removed or becomes unitary giving raise to another filtering step (by unit propagation).

Efficient and scalable algorithms for SAT, that were developed over the last decade, have contributed to dramatic advances in the ability to automatically solve problem instances involving tens of thousands of variables and millions of constraints. That is why, we have chosen to encode a query as a CNF and then use a SAT solver to answer it.

\subsection{Variables and Encoding of Partitioning Constraints}

The data analyst formulates his queries by using the constraint-based language introduced above. Let $\mathcal{T}$ be the dataset to be proceeded. The CNF encoding a query $q_{i}$ is the conjunction of the CNFs of the constraints involved in $q_{i}$.

Each unknown cluster is modeled using $m$ boolean variables $T_{t, j}$ such that $\left(T_{t, j}=\right.$ True $)$ iff transaction $t$ belongs to cluster $j$. A cluster is referenced by its index between 1 and $k$.

- coverTransactions $\left(\left[X_{1}, \ldots, X_{k}\right]\right)$ ensures that each transaction $t \in \mathcal{T}$ belongs to at least one cluster. So, for each $t$, there exists at least one cluster $X_{j}$ s.t. $t \in X_{j}$.

$$
\bigwedge_{t \in \mathcal{T}}\left(\bigvee_{j \in[1 . . . k]} T_{t, j}\right)
$$

- noOverlapTransactions $\left(\left[X_{1}, \ldots, X_{k}\right]\right)$ ensures that each transaction $t \in \mathcal{T}$ belongs to at most one cluster. So, a transaction $t$ belongs to (at least) two clusters iff there exist $X_{i}$ and $X_{j}$ s.t. $\left(t \in X_{i}\right) \wedge\left(t \in X_{j}\right)$, i.e. $\bigvee_{1 \leq i<j \leq k}\left(T_{t, i} \wedge\right.$ $\left.T_{t, j}\right)$. So, the negation must hold for each transaction $t$.

$$
\bigwedge_{t \in \mathcal{T}}\left(\bigwedge_{1 \leq i<j \leq k}\left(\neg T_{t, i} \vee \neg T_{t, j}\right)\right)
$$

- isNotEmpty $\left(X_{j}\right)$ ensures that there exists at least one transaction $t \in \mathcal{T}$ that belongs to cluster $X_{j}$ and is modeled by the clause: $\vee_{t \in \mathcal{T}} T_{t, j}$

- canonical $\left(\left[X_{1}, \ldots, X_{k}\right]\right)$ ensures that, for all $i$ s.t. $1 \leq i<k$, cluster $X_{i}$ is less than cluster $X_{i+1}$. This constraint is encoded using a binary comparator.

A constraint coverItems $\left(\left[X_{1}, \ldots, X_{k}\right]\right)$ (resp. noOverlapItems $\left.\left(\left[X_{1}, \ldots, X_{k}\right]\right)\right)$ is encoded in the same way as a constraint coverTransactions $\left(\left[X_{1}, \ldots, X_{k}\right]\right)$ (resp. noDverlapTransactions $\left.\left(\left[X_{1}, \ldots, X_{k}\right]\right)\right)$. 


\subsection{Encoding mustLink and cannotLink Constraints}

mustLink $\left(t_{1}, t_{2}\right)$ ensures that the transactions $t_{1}$ and $t_{2}$ belong to the same cluster. So, for each $j \in[1 . . k], T_{t_{1}, j} \Leftrightarrow T_{t_{2}, j}$.

$$
\bigwedge_{1 \leq j \leq k}\left(\neg T_{t_{1}, j} \vee T_{t_{2}, j}\right) \wedge\left(T_{t_{1}, j} \vee \neg T_{t_{2}, j}\right)
$$

In the same way, cannotLink $\left(t_{1}, t_{2}\right)$ is encoded as:

$$
\bigwedge_{1 \leq j \leq k}\left(\neg T_{t_{1}, j} \vee \neg T_{t_{2}, j}\right) \wedge\left(T_{t_{1}, j} \vee T_{t_{2}, j}\right)
$$

So each transaction level constraint is encoded using $(2 \times k)$ binary clauses.

\subsection{Encoding Threshold Constraints Using Sorting Networks}

Threshold constraints are directly modeled using cardinality constraints. So, $\operatorname{size}\left(X_{j}\right) \geq \delta_{1}$ is modeled as $\#\left(T_{1, j}, T_{2, j}, \ldots, T_{m, j}\right) \geq \delta_{1}$. This cardinality constraint states that at least $\delta_{1}$ variables $T_{t, j}$ must be assigned to True. Other threshold constraints involving function symbols are encoded in the same way.

Several efficient CNF encodings of cardinality constraints have been proposed 118. Cardinality constraints are encoded thanks to unary adders in order to perform filtering by unit propagation. But, such encodings require a quadratic number of clauses [1] or they depend on the value of the threshold [18. Moreover, for clustering, or other data mining tasks, thresholds can have rather large values, so the size of such encodings can quickly become prohibitive.

We used sorting networks to encode threshold constraints because the size of the resulting encoding does not depend on the value of the threshold. Moreover, using sorting networks to implement cardinality constraints preserves arcconsistency 11. The odd-even Batcher sort [4] proved to be very efficient compared to other encodings of cardinality constraints [1].

\subsection{Transitive Inference of mustLink and cannotLink Constraints}

Let $G=(V, E)$ be the mustLink graph where $V=\mathcal{T}$. There is an edge between $t_{i}$ and $t_{j}$ iff there exists a constraint mustLink $\left(t_{i}, t_{j}\right)$ 320. Let $C C_{1}$ and $C C_{2}$ be two connected components of $G$. (i) If there exists a constraint mustLink $\left(t_{1}, t_{2}\right)$ with $t_{1} \in C C_{1}$ and $t_{2} \in C C_{2}$, then we can infer the constraints mustLink $(x, y)$ for all $x \in C C_{1}$ and $y \in C C_{2}$. Contrary to mustLink, cannotLink is not an equivalence relation, but (ii) If there exists a constraint cannotLink $\left(t_{1}, t_{2}\right)$ with $t_{1} \in C C_{1}$ and $t_{2} \in C C_{2}$, then we can infer the constraints cannotLink $(x, y)$ for all $x \in C C_{1}$ and $y \in C C_{2}$.

Such entailments are usually performed by adding all the inferred mustLink and cannotLink constraints 320]. But, using our SAT encoding (see Section 4.3), there is no need to perform those addings: all inferred constraints are implicitly stated and will be taken into account by the SAT solver. In fact, mustLink and cannotLink constraints are encoded using equivalence between boolean variables (see Section 4.3). 
Table 3. Dataset's characteristics

\begin{tabular}{|c|c|c|c|c|c|}
\hline datase & Australia & & & & \\
\hline$m$ & 653 & 8124 & 336 & 630 & 101 \\
\hline$n$ & 125 & 119 & 36 & 50 & 36 \\
\hline density & 0.40 & 0.19 & 0.48 & 0.32 & 0.44 \\
\hline
\end{tabular}

\subsection{Ensuring Completeness}

Given a CNF, SAT solvers either find one instantiation (and only one) for the variables evaluating the formula to True, or prove there is no such an instantiation. In order to ensure the completeness of our approach, restarts are performed. Let $\mathcal{F}$ be the CNF modeling a query $q$. Resolution begins with $\mathcal{F}$. Then, after having obtained the $i$-th solution $s_{i}$, its negation $\neg s_{i}$ is added to the (current) CNF and resolution is restarted in order to look for another solution. The process ends when a failure occurs, i.e. when all solutions have been found. Using restarts may seem too naive, but in practice is efficient enough. As CNFs contain much binary clauses, filtering by unit propagation is very effective (see experiments performed in Section 5).

\section{Experiments}

The goal of the experiments is to provide better insights on our constrained clustering method according to several constraints and datasets. We used the MiniSat 1 solver [10] to implement our method. We performed experiments on several datasets from the UCI repository 2 (see Table 3). Experiments were conducted on a Core2Duo E8400 (2.83GHz) with 4GB of RAM. For each experiment, we report the CPU-times needed to compute the first and the first ten solutions 3 according to the required number of clusters $k$.

We used the Hamming distance 4 between transactions. The maximum diameter $\alpha$ has been set to $n / 2$ (if two transactions differ more than $50 \%$, they cannot belong to the same cluster) and the minimum separation $\beta$ to $n / 20$ (if two transactions differ less than $5 \%$, they must belong to the same cluster).

Fig. 1 reports CPU-times needed to compute the first and the first ten solutions for query $q_{1}$ (see Section 3.2). For each dataset, the first ten solutions (if there exist) are obtained very quickly, even for the dataset Mushroom which is the largest one.

Fig. 2 reports CPU-times needed to compute the first solution for balanced clustering (query $q_{3}$, Section $[3.2$ ). The balancing ratio $\Delta$ has been set to $10 \%$ (Fig.2left) and to 20\% (Fig.2 right). Even with additional threshold constraints, our approach is still efficient. Note that with such restrictive thresholds, few queries have a solution.

${ }^{1}$ http://minisat.se/

${ }^{2}$ http://www.ics.uci.edu/ mlearn/MLRepository.html

${ }^{3}$ Symbol '-' denotes the absence of solution for a query.

${ }^{4}$ Any distance can be used since distance is only used to state mustLink and cannotLink constraints. 


\begin{tabular}{l|ccccc}
\hline dataset & $k=4$ & $k=8$ & $k=12$ & $k=16$ & $k=20$ \\
\hline Australian & - & 0.02 & 0.25 & 0.89 & 1.59 \\
Mushroom & 0.96 & 1.38 & 3.94 & 6.64 & - \\
P.-Tumor & - & 0.03 & 0.08 & 0.12 & 0.13 \\
Soybean & 0.01 & 0.03 & 0.12 & 0.16 & - \\
Zoo & 0.01 & 0.01 & 0.02 & 0.03 & 0.03 \\
\hline
\end{tabular}

\begin{tabular}{ccccc}
\hline$k=4$ & $k=8$ & $k=12$ & $k=16$ & $k=20$ \\
\hline- & 0.05 & 0.31 & 0.96 & 1.70 \\
1.28 & 1.53 & 5.38 & 8.24 & - \\
- & 0.03 & 0.11 & 0.15 & 0.17 \\
0.02 & 0.05 & 0.14 & 0.18 & - \\
0.01 & 0.01 & 0.03 & 0.04 & 0.04 \\
\hline
\end{tabular}

Fig. 1. $\left(q_{1}\right)$ Time in s. to obtain the $1^{\text {st }}$ sol. (left) and the first ten sol. (right)

\begin{tabular}{l|ccccc}
\hline dataset & $k=4$ & $k=8$ & $k=12$ & $k=16$ & $k=20$ \\
\hline Australian & - & 19.7 & 27.46 & 45.23 & 180.5 \\
Mushroom & 16.9 & - & - & - & - \\
P.-Tumor & - & 1.06 & 2.22 & 6.16 & 6.05 \\
Soybean & - & - & - & - & - \\
Zoo & 0.01 & 0.09 & - & - & - \\
\hline
\end{tabular}

\begin{tabular}{ccccc}
\hline$k=4$ & $k=8$ & $k=12$ & $k=16$ & $k=20$ \\
\hline- & 7.20 & 13.10 & 46.15 & 69.76 \\
19.6 & - & - & - & - \\
- & 0.91 & 3.30 & 3.88 & 6.08 \\
- & - & - & - & - \\
0.03 & 0.10 & - & - & - \\
\hline
\end{tabular}

Fig. 2. $\left(q_{3}\right)$ Time in s. for the $1^{\text {st }}$ sol. with $\Delta=10 \%$ (left) and with $\Delta=20 \%$ (right)

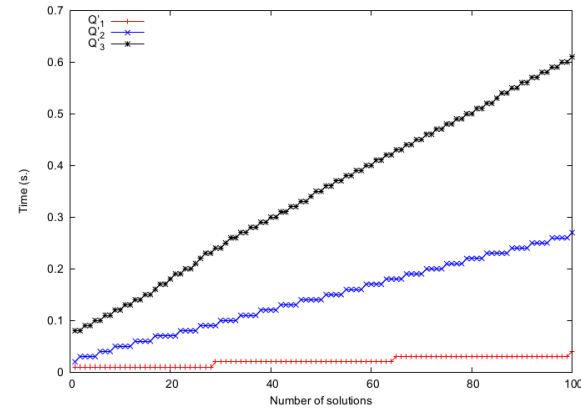

Fig. 3. Time for Zoo $(k=6)$

\begin{tabular}{|cc|rrr|}
\hline Australian & $(k=2)$ & $(k=6)$ & $(k=10)$ \\
\hline$q_{1}$ \#literal & 2.6 & 10.4 & 18.2 \\
& \#clause & 29.8 & 107.8 & 196.3 \\
& $r \%$ & 86.9 & 81.2 & 83.93 \\
\hline$q_{2}$ \#literal & 98.8 & 299.2 & 499.5 \\
& \#clause & 318.7 & 974.5 & 1640 \\
& $r \%$ & 68.6 & 68.3 & 68.5 \\
\hline$q_{3}$ \#literal & 98.8 & 299.2 & 499.5 \\
& \#clause & 318.7 & 974.5 & 1640 \\
& $r \%$ & 68.6 & 68.3 & 68.5 \\
\hline
\end{tabular}

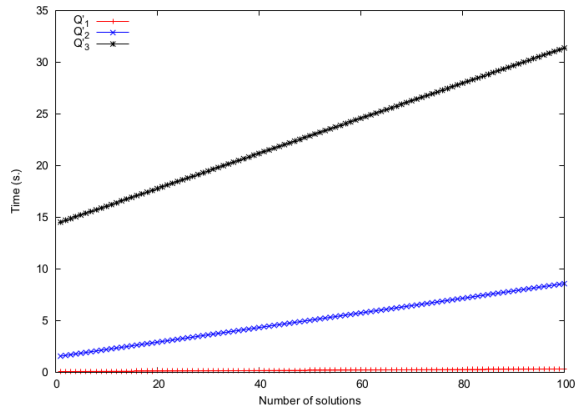

Fig. 4. Time for Australian $(k=6)$

\begin{tabular}{|c|rrr|}
\hline Mushroom & $(k=2)$ & $(k=6)$ & $(k=10)$ \\
\hline$q_{1}$ \#literal & 32.4 & 129.9 & 227.4 \\
\#clause & 1141 & 3651 & 6291 \\
$r \%$ & 95.7 & 93.1 & 92.9 \\
\hline$q_{2}$ \#literal & 1343 & 4062 & 6781 \\
\#clause & 5075 & 15452 & 25960 \\
$r \%$ & 73.2 & 72.9 & 73.1 \\
\hline$q_{3}$ \#literal & 1343 & 4062 & 6781 \\
\#clause & 5075 & 15452 & 25960 \\
$r \%$ & 73.2 & 72.9 & 73.1 \\
\hline
\end{tabular}

Fig. 5. Number of literals and clauses for the three queries (in thousands)

Fig. 3 depicts the CPU-times according to the number of solutions for dataset Zoo with $k=6$. A curve is associated to each of the three queries $q_{1}$ (red), $q_{2}$ with $\delta=m / 10$ (blue) and $q_{3}$ with $\Delta=20 \%$ (black). These curves seem to be quasi-linear. All the three queries mainly involve mustLink and cannotLink constraints which are encoded using binary clauses. As soon as a transaction is assigned to a cluster, 
a lot of deductions are performed using unit propagation and transitive inferences (see Section 4.5). Nevertheless, further investigations are required to confirm this result. Fig. 4 provides similar results for dataset Australian with $k=6$.

Fig. 5 reports the size of the encodings of queries $q_{1}, q_{2}$, and $q_{3}$ for datasets Australian and Mushroom, as well as the ratio $(r)$ of binary clauses constituting the CNF. The encoding of a query could be large (several millions of clauses), but the ratio $r$ always remains very high. The size of $q_{2}$ is similar as the size of $q_{3}$ since only the bounds of the cardinality constraints are changed (see Section 4.4). To sum up, these experiments show that SAT solvers allow to solve efficiently this clustering task. They can find the first solutions (or prove there is none) in affordable times, even for medium scale size datasets as Mushroom. However, most of the clustering queries need threshold constraints which require more computational efforts.

\section{Related Work}

SAT for Clustering. Constraints on clusters (e.g., Maximum Diameter and Minimum Separation) into the k-means clustering algorithm [16] have been introduced by [7. A formal complexity analysis of constraints on transactions and clusters is performed in 8]. Davidson and al. have proposed the first approach using SAT for clustering [9], but it deals only with a strong limited setting $(k=2)$. The authors show how constraints both on transaction and cluster levels can be modeled and solved as instances of the 2-SAT problem. Gilpin and Davidson consider hierarchical constrained clustering and describe how dendograms can be modeled and solved as instances of the Horn-SAT problem [13. We have also used SAT to implement primitives of our constraint-based language [17.

SAT for Mining Patterns. a SAT approach for enumerating all frequent patterns with wildcards in a given sequence has been proposed in [6].

\section{Conclusion and Future Work}

We have proposed a constrained clustering approach handling a large set of constraints. Solving is performed thanks to a SAT encoding for clustering and we have described how queries can be solved by taking benefit from features of SAT solvers. Experiments performed using MiniSat show the feasibility and the effectiveness of our approach. An insight of our work is that when a certain clustering task is modeled, many variants of that task can be modeled as well, changing or adding a few constraints is sufficient to allow this to happen.

As future work, we want to enrich our constraint-based language with further primitives to determine and/or constrain the cluster center locations. We also want to improve the current encoding (e.g., defining labeling orderings, exploiting backdoors, nogoods... ). Another challenge is to propose an alternative encoding consuming less space and yet having relevant properties for an efficient solving. We want to conduct an in-depth study of the scalability of the approach to larger values of $k$ and larger datasets. Finally, another promising direction is to integrate optimization criteria in our framework. 


\section{References}

1. Bailleux, O., Boufkhad, Y.: Efficient CNF Encoding of Boolean Cardinality Constraints. In: Rossi, F. (ed.) CP 2003. LNCS, vol. 2833, pp. 108-122. Springer, Heidelberg (2003)

2. Banerjee, A., Ghosh, J.: Scalable clustering algorithms with balancing constraints. Data Min. Knowl. Discov. 13(3), 365-395 (2006)

3. Basu, S., Davidson, I., Wagstaff, K.L.: Constrained Clustering: Advances in Algorithms, Theory, and Applications. Chapman \& Hall (2008)

4. Batcher, K.E.: Sorting networks and their applications. In: AFIPS Spring Joint Computing Conference. AFIPS Conference Proceedings, vol. 32, pp. 307-314. Thomson Book Company, Washington, D.C (1968)

5. Berkhin, P.: Survey of clustering data mining techniques. Technical report, Accrue Software, San Jose, CA, USA (2002)

6. Coquery, E., Jabbour, S., Sais, L.: A constraint programming approach for enumerating motifs in a sequence. In: Workshop on Declarative Pattern Mining, ICDM 2011, Vancouver, Canada, pp. 1091-1097 (December 2011)

7. Davidson, I., Ravi, S.: Clustering with constraints: Feasibility issues and the kmeans algorithm. In: SDM (2005)

8. Davidson, I., Ravi, S.: Using instance-level constraints in agglomerative hierarchical clustering: theoretical and empirical results. Data Min. Knowl. Discov. 18(2), 257-282 (2009)

9. Davidson, I., Ravi, S., Shamis, L.: A SAT-based framework for efficient constrained clustering. In: SDM, pp. 94-105. SIAM (2010)

10. Eén, N., Sörensson, N.: An Extensible SAT-solver. In: Giunchiglia, E., Tacchella, A. (eds.) SAT 2003. LNCS, vol. 2919, pp. 502-518. Springer, Heidelberg (2004)

11. Eén, N., Sörensson, N.: Translating pseudo-boolean constraints into SAT. JSAT 2(1-4), 1-26 (2006)

12. Fisher, D.H.: Knowledge acquisition via incremental conceptual clustering. Machine Learning 2(2), 139-172 (1987)

13. Gilpin, S., Davidson, I.: Incorporating SAT solvers into hierarchical clustering algorithms: an efficient and flexible approach. In: KDD 2011, pp. 1136-1144 (2011)

14. Guns, T., Nijssen, S., De Raedt, L.: Itemset mining: A constraint programming perspective. Artif. Intell. 175(12-13), 1951-1983 (2011)

15. Khiari, M., Boizumault, P., Crémilleux, B.: Constraint Programming for Mining nary Patterns. In: Cohen, D. (ed.) CP 2010. LNCS, vol. 6308, pp. 552-567. Springer, Heidelberg (2010)

16. MacQueen, J.B.: Some methods for classification and analysis of multivariate observations. In: Proceedings of the 5th Berkeley Symposium on Mathematical Statistics and Probability, vol. 1, pp. 281-297. University of California Press (1967)

17. Métivier, J.-P., Boizumault, P., Crémilleux, B., Khiari, M., Loudni, S.: A constraint-based language for declarative pattern discovery. In: 27th Annual ACM Symposium on Applied Computing (SAC 2012), March 2012, pp. 438-444 (2012)

18. Sinz, C.: Towards an Optimal CNF Encoding of Boolean Cardinality Constraints. In: van Beek, P. (ed.) CP 2005. LNCS, vol. 3709, pp. 827-831. Springer, Heidelberg (2005)

19. Tung, A.K.H., Han, J., Lakshmanan, L.V.S., Ng, R.T.: Constraint-Based Clustering in Large Databases. In: Van den Bussche, J., Vianu, V. (eds.) ICDT 2001. LNCS, vol. 1973, pp. 405-419. Springer, Heidelberg (2000)

20. Wagstaff, K., Cardie, C.: Clustering with instance-level constraints. In: ICML 2000, pp. 1103-1110. M. Kaufmann (2000) 